\title{
Pathomorphogenesis in goats with Coccidiosis
}

\author{
V.M. Usevich*, N.I. Zhenikhova, M.N. Drozd, and L. Banda \\ Ural State Agrarian University, Yekaterinburg, Russia
}

\begin{abstract}
The problem of the spread of coccidiosis (eimeriosis) in animals is still an urgent problem for veterinary specialists around the world. In this regard, the aim of the study was to study the most complete pathomorphological picture of this disease in goats. The research material was the fallen kids of the Alpine breed. The research was carried out on the basis of one of the goat breeding farms in the Sverdlovsk region. Diagnostics was performed according to clinical, laboratory and pathomorphological studies. Research results. The article describes the morphological picture of pathological changes in the parenchymal and target organs. The scientific novelty lies in the fact that a morphological assessment of changes in target organs, parenchymal organs that provide detoxification function in the body, in the organs of the immune system and in parenchymal organs that perform vital functions in the body of blood circulation and gas exchange has been carried out.
\end{abstract}

\section{Introduction}

Diseases of the digestive system bring tangible economic damage to all livestock enterprises, which consists of losses in the meat and dairy productivity of animals, the quality of the livestock products obtained, a decrease in feed conversion and death of animals. These diseases are of the most varied nature. These include diseases of a viral, bacterial and parasitic nature. Many of them belong to the group of zoonotic diseases, and some to the group of zooanthroponoses.

One of these diseases is coccidiosis (eimeriosis) of ruminants, which occurs when infected with protozoa, which belong to the Sporozoa class. The prevalence of coccidiosis diseases in various animal species has a global scale and ranges from 33 to $100 \%$, therefore, this problem has attracted the attention of veterinarians around the world, where people are not a vegetarian diet.

Eimeriosis (coccidiosis) affects many species of animals, but each species has its own pathogens and their species composition is different, while the disease can be provoked by several Eimeria at once, while the disease proceeds as a mixed infection. [26, 27]

Our previous studies have shown that the most common eimeriosis is caused by two or three dissimilar Eimeria.

Eimeria - belongs to the Protozoa group. According to the literature, the pathogen affects the epithelial cells of the intestinal mucosa and causes a violation of the processes of digestion and absorption. The developmental cycle of the parasite proceeds cyclically intra-

\footnotetext{
* Corresponding author: vus5@yandex.ru
} 
enterocyte and intra-enteric with the release of oocysts into the intestinal cavity. As a result, the intestinal mucous membranes become inflamed, destroyed and extensive erosions appear, decreased intestinal barrier functions. Intoxication, diarrhea, impaired waterelectrolyte metabolism develops.

Timely differential diagnosis contributes to the timely adequate treatment of such animals [26, 27].

Aim and Objectives

In connection with all of the above, the goal was set: to study the Pathomorphogenesis of internal organs in kids with eimeriosis.

To achieve this goal, the following tasks were set:

to determine the macropathological changes in coccidiosis in kids;

to determine micromorphological changes in the parenchymal organs of kids with eimeriosis.

\section{Methodology and research methods}

To accomplish the set objectives, studies were carried out on Alpine goats that died from eimeriosis in the conditions of an industrial goat breeding economy in the Middle Urals.

The main direction of the goat farm is the production of cheese. On the farm, diseases of young animals, accompanied by dyspeptic disorders, are noted, and these disorders are recorded not in the early postnatal period in newborn animals, but already in grown young animals, which are clearly manifested during regrouping and transfer of animals from one room to another, sometimes these symptoms were noted when changing the diet. In connection with the above, experimental studies were carried out to diagnose eimeriosis in young ruminants. Laboratory studies were performed at the Department of Infectious and Non-infectious Pathology of the Ural State Agrarian University and the Sverdlovsk Regional Veterinary Laboratory.

Before the start of the research, a full clinical study of animals was carried out, then scatological and helminthic studies, to exclude bacteriological infections, bacteriological studies were carried out in the bacteriological department of the regional veterinary laboratory. Samples of feces taken from the rectum individually served as material for coprological and helminth cyst-ovoscopic studies. Fecal samples were examined according to standard methods: the native smear method and the Fülleborn's method, according to (Interstate Council of Standardization and Certification 54627-2011). Microscopy of smears was performed using a Micromed-1 microscope, and photographic recording using a Levenhuk $\mathrm{C}$ series C300 digital camera. The digital data was processed statistically.

During the postmortem examination of the fallen kids, a macro picture of the pathological changes was described, and material was taken for histological examination. For this, pieces of organs $\left(1 \mathrm{~cm}^{3}\right)$ in size were taken. The pieces were placed in a $10 \%$ solution of neutral formalin, fixed, then subjected to dehydration through alcohols of increasing concentration, then embedded in paraffin, then sections were prepared and stained with hematoxylin and eosin. After that, they were examined under a light microscope and photographed. For this, a MiCmed-1 microscope and a Levenhuk C series C300 digital camera for a microscope were used.

During the pathological autopsy, samples of internal organs were taken and histologically examined: a booklet, a mesh, intestines, liver, kidney, spleen, lung.

All studies, diagnostics and treatment of animals were carried out in accordance with Directive 2010/63 / EU of the European Parliament and the Council of the European Union "On the protection of animals used for scientific purposes."

Laboratory studies of the biomaterial were carried out on the basis and in accordance with the requirements of the regulations, "The list of regulatory documents permitted for use in 
state veterinary laboratories in the diagnosis of diseases of animals, fish, bees, as well as control of the safety of raw materials of animal and vegetable origin", approved by the Federal Service for Veterinary and Phytosanitary Supervision from July 30, 2009, No. 02$03-48$ / 563.3.

\section{Results}

In the study of coprogram and feces for helminthic Ovo cystoscopy in three-week-old kids, associations of Eimeria of two species prevailed - Eimeria arloingi (68\%), Eimeria ninaekohlyakimovae (32\%) (Fig. 1).

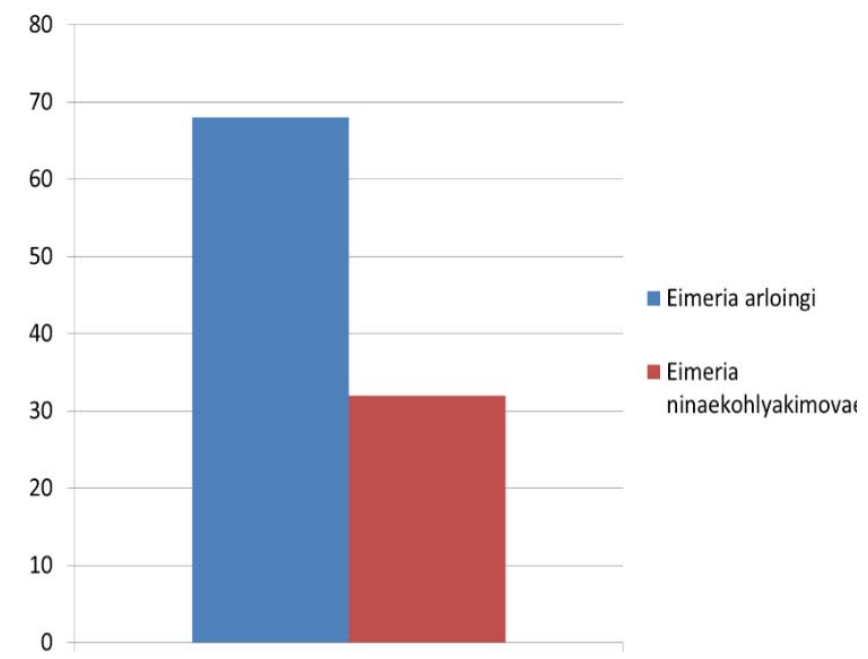

Fig. 1. Species composition of Eimeria oocysts in kids 3 weeks of age.

Despite the treatment of Eimeriosis with coccidiostats carried out on the farm, 3 kids out of 10 examined died.

At postmortem examination of the bodies of the fallen kids, a macroscopic examination of the abomasum and reticulum revealed catarrhal and catarrhal-fibrinous inflammation. The leaflets of the abomasum are compacted and thickened, covered with viscous mucus, microscopically, the submucous layer is in a state of edema (Fig. 2). In the mesh, the mucous membrane is covered with thick, viscous mucus and fibrin threads (Fig. 3).

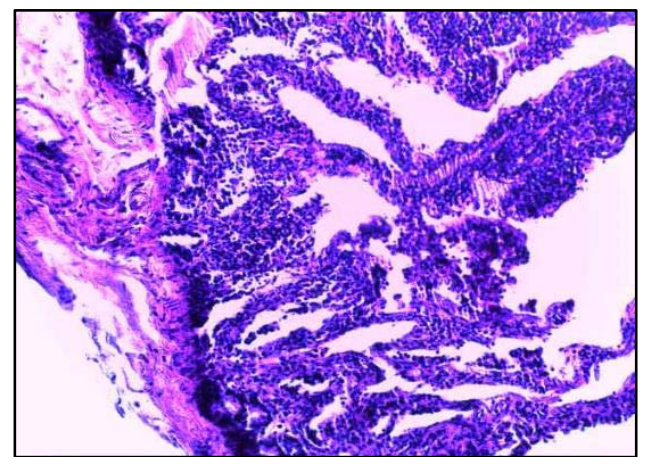

Fig. 2. Abomasum. Catarrhal inflammation of the mucous membrane, edema of the submucosa. Magnification x 200 .

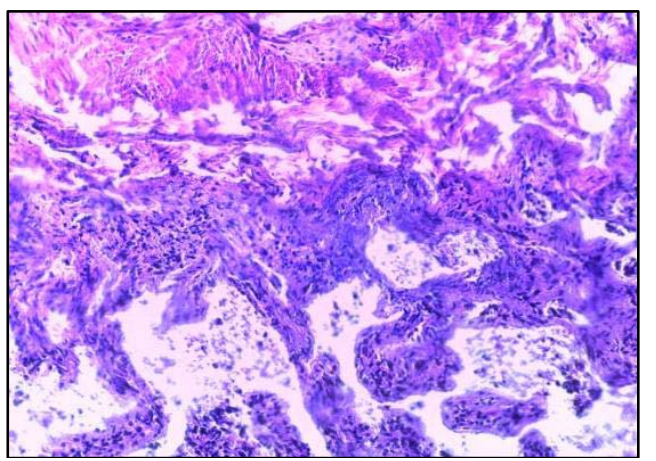

Fig. 3. Reticulum. Catarrhal fibrinous inflammation of the mucosa, edema of the submucosa. Magnification x400 
Macroscopic examination of the small and large sections of the intestine revealed an increase in volume and hyperemia of the wall. In the folds of the mucous membrane, small punctate hemorrhages and erosion are noticeable. The mucous membrane is covered with a thick layer of mucus. Lymphoid follicles protrude above the mucous membrane and are up to a match head, grayish from the surface, visible better from the side of the serous membrane, located deep in the thickness of the intestinal wall. In the small intestine, grayish-white nodules the size of a grain of millet are visible.

The mucous membrane of the large intestine has total or multiple local catarrhal or catarrhal-hemorrhagic inflammation. Ulcerative lesions are often found on the surface of the mucous membrane of the rectum and cecum. Intestinal contents ranging from dark chocolate to reddish brown.

Histological examination in the small intestine showed shortening, flattening of the villi with absent or reduced glycocalyx and atrophy of microvilli. Intra- and extracellular edema was noted in the cytoplasm of enterocytes. In the mucous and submucosal layers of the intestinal wall diffuse polymorphic cell infiltration by eosinophils, histiocytes, macrophages, fibroblasts and neutrophils (Fig. 4). They contribute to the development of productive inflammation at the site of the formation of parasitic granulomas. Hemorrhage around damaged vessels. The mucous membrane of the duodenum is thickened, hyperemic, inflamed, edematous, has hemorrhages, the villi pattern is smoothed. In the lumen of the intestine there is a lot of viscous mucus of a grayish color with streaks of blood.

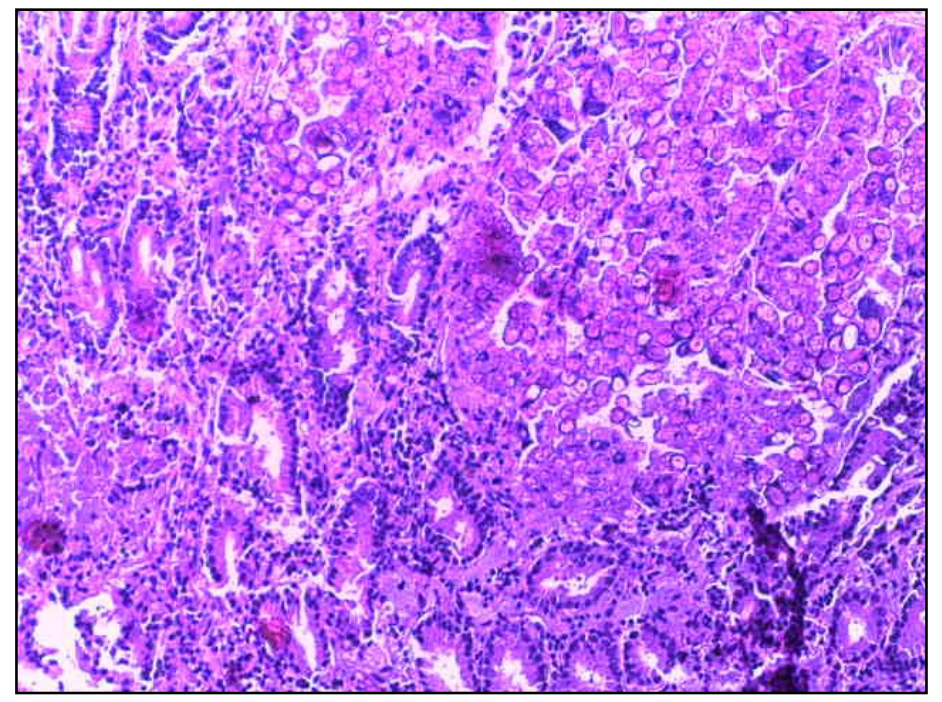

Fig. 4. Wall of the duodenum. Diffuse polymorphic cell infiltration. Mag x200.

In the large intestine, gut wall edema and hemorrhages were macroscopically observed. The mucous membrane of the large intestine was dark red with petechial hemorrhages. The wall of the large intestine loops is increased in volume. The cecum in a state of hemorrhagic inflammation. The intestinal cavity contains hemorrhagic-fibrinous exudate. Microscopically in the contents of the intestine - enterocytes, erythrocytes, leukocytes and Eimeria of all stages of development.

Macroscopic examination revealed hepatomegaly, the surface is colored nonuniformly, in the form of whitish spots with indistinct borders and nodules ranging in size from millet grain to a medium pea on a brown background. The nodules in the cut have a thick creamy content. The liver is of a flabby consistency; on the cut there are isolated foci of an offwhite or yellowish color, oval or round, oriented mainly towards the bile ducts. They are 
isolated from the rest of the liver parenchyma by connective tissue. The bile ducts are dilated. The bile is thick. Microscopy of a smear from the contents of the nodules revealed many Eimeria oocysts.

Microscopic examination in the liver showed a violation of the characteristic structure, the absence of a beam structure, signs of granular degeneration of hepatocytes (Fig. 5). In the endothelium of blood vessels - erosive damage and focal hemorrhages. In the parenchyma, there are areas of hepatocyte necrosis (Fig. 6).

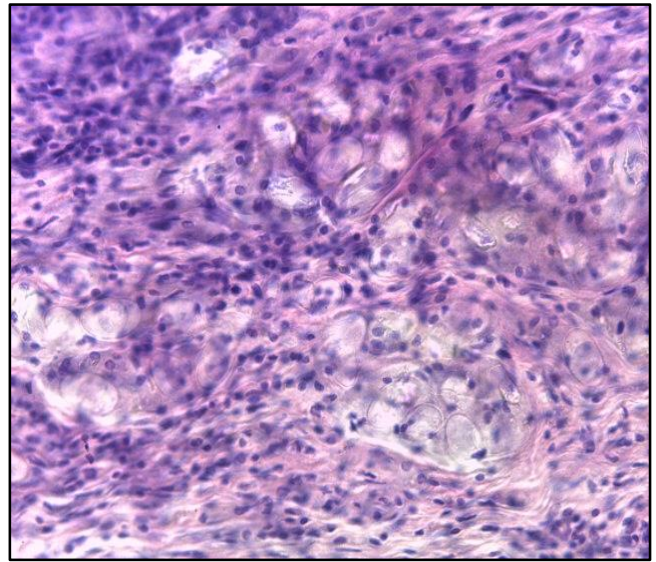

Fig. 5. The liver. Accumulation of oocysts in the liver parenchyma. Mag x400

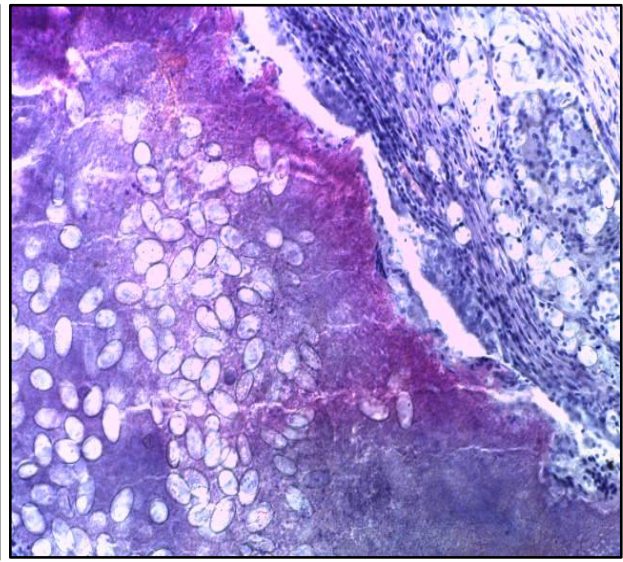

Fig. 6. Liver. Chalicosis with a necrotic center and an accumulation of oocysts $\mathrm{x} 400$.

Microscopy of histological sections in the lungs recorded congestion: hyperemia, edema, as well as emphysema and bronchiectasis (Fig. 7). Some have foci of pneumonia and pleurisy (Fig. 8). In the bronchi - desquamation of the epithelium and mucous dystrophy.

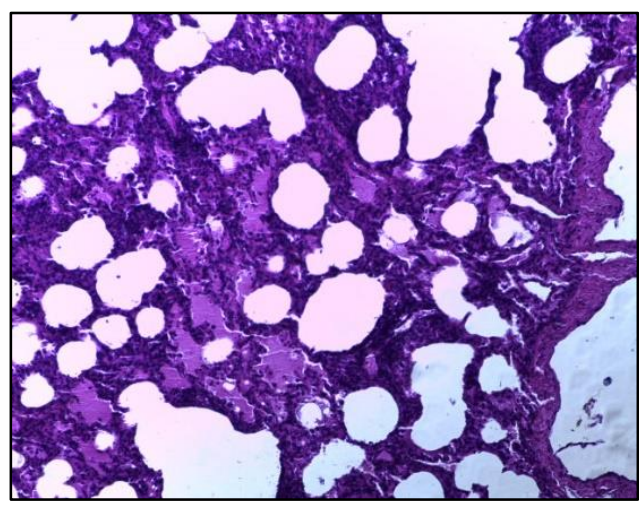

Fig. 7. Lung. Perivascular edema, bronchiectasis. Mag x200.

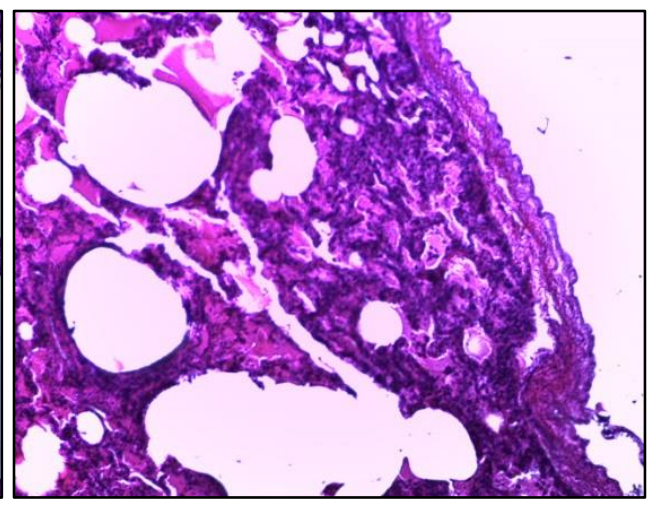

Fig. 8. Lung. Pleuritis, ectasia in the lung. Mag $\mathrm{x} 200$.

Histological examination of the kidneys revealed: perivascular edema (Fig. 9), granular degeneration of the convoluted tubules, focal necrosis and glomerulonephritis (Fig. 10). 


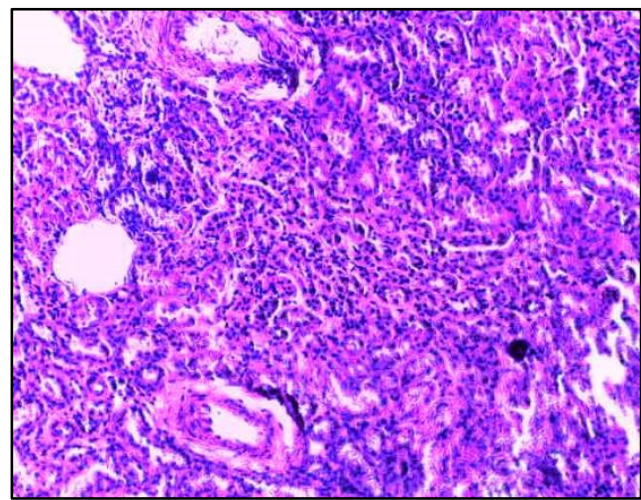

Fig. 9. Kidney. Perivascular edema. Magnification x200.

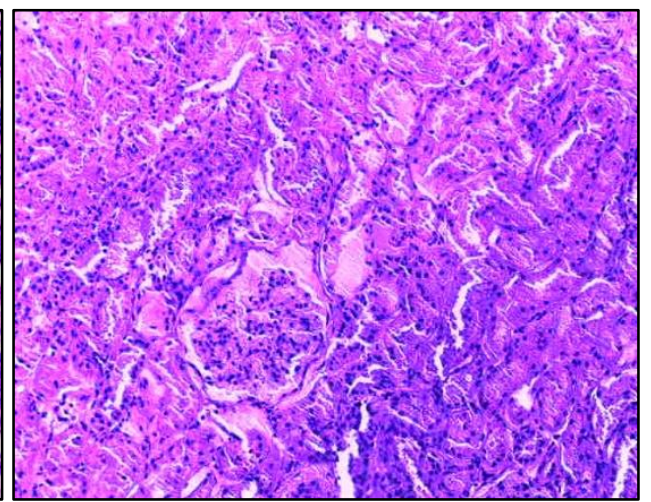

Fig. 10. Kidney. Glomerulonephritis. Magnification x 400 .

Macroscopic examination of the spleen revealed its compaction, and histological examination revealed thickening of the capsule (Fig. 11), proliferation of connective tissue, reduction of lymphoid follicles, vascular obstruction, and perivascular edema (Fig. 12).

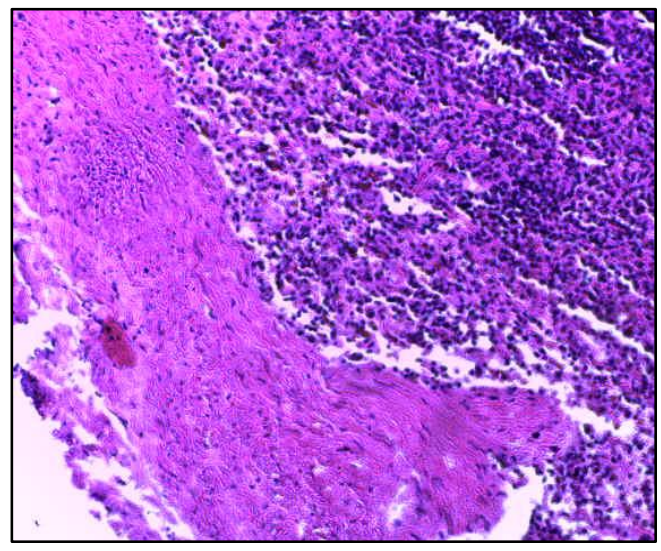

Fig. 11. Spleen. Thickening of the capsule. Magnification x200.

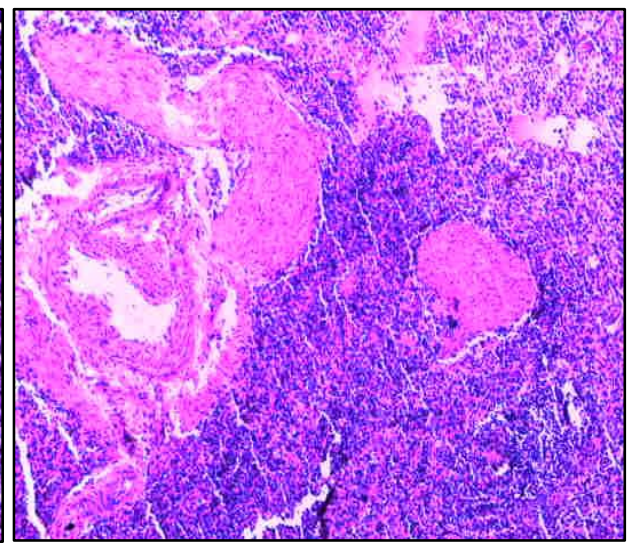

Fig.12. Spleen. Follicular reduction, vascular obstruction and perivascular edema. Magnification x 200 .

As a result of the conducted micromorphological studies of parenchymal organs, in which there were macroscopic signs of lesion, it is possible to state a severe pathological condition of kids with eimeriosis, accompanied by suppression of the immune system and the development of a pathological process with damage to most parenchymal organs.

\section{Discussion of research results}

When analyzing the results of the study, it can be noted that with eimeriosis of goats, total damage to the cardiovascular system develops, corresponding to a strong degree of intoxication of the body. Immunosuppression and immune dystrophy are noted against the background of intoxication. Inflammatory and dystrophic-necrotic processes at the sites of introduction of the parasite in the digestive system, excretion and detoxification, inflammatory-compensatory - in the cardiovascular and respiratory systems. 


\section{Conclusions}

As a result of the analysis of the research carried out, the following conclusions can be drawn:

1. Coccidiosis in kids at three weeks of age was caused by mixed invasions of Eimeria of two species - Eimeria arloingi (68\%), Eimeria ninaekohlyakimovae (32\%).

2. With eimeriosis in kids, macro-micromorphological changes are noted in the digestive organs, the detoxification of organs - the liver, the organs of excretion of metabolic products and toxins - the kidneys, the organs of the immune system and the organs of life support - the heart and lungs.

3. As a result of the conducted micromorphological studies of parenchymal organs, in which there were macroscopic signs of lesion, a severe pathological condition of kids with eimeriosis, accompanied by immunosuppression and the development of a pathological process with damage to most of the parenchymal organs, can be observed.

\section{Conflict of interest}

The authors of the article declare that the information provided does not contain any conflicts of interest.

\section{References}

1. D.A. Zhelyabovskaya [et al.], Bulletin of KrasGAU, 11, 27-33 (2017)

2. E.V. Pechura, A.P. Poryvaeva, I.M. Sazhaev, N.A. Kutkina, Proceedings of the Kuban State Agrarian University, 2 (83), 187-194 (2020)

3. I.M. Sazhaev, E.V. Pechura, Epizootological monitoring of parasitoses in cattle on the territory of the Sverdlovsk region, Actual problems and issues of veterinary medicine and biotechnology in modern conditions of development: materials of the regional scientific and practical interdepartmental conference, Samara, 151-155 (2016)

4. N.A. Vereshchak, A.P. Poryvaeva, E.V. Pechura [and others], Issues of legal regulation in veterinary medicine, 4, 16 (2016)

5. I.M. Donnik, I.A. Shkuratova, Herald of the Russian Academy of Sciences, 87 (2), 139-142 (2017)

6. S.T.N. Keeton, C.B. Navarre, Veterinary Clinics of North America: Food Animal Practice, 34 (1), 201-208 (2018) DOI: 10.1016 / j.cvfa.2017.10.009

7. M.A. Taylor, R.L. Coop, R.L. Wall, Veterinary parasitology, 1032 (Ames (IA), Wiley Blackwell, 2016)

8. S. Schoenian, Coccidiosis: deadly scourge of lambs and kids, Maryland small ruminant page (2016) URL: http://www.sheepandgoat.com/coccidiosis.

9. Coccidiosis, Calfology, (2019) URL: http://calfology.com/library/wiki/coccidiosis DOI: 10.13140 / RG.2.2.17932.62088

10. P. Gibbons, D. Love, T. Craig, et al., Veterinary Parasitology, 218, 1-4 (2016)

11. W.I. Mohamaden, N.H. Sallam, E.M. Abouelhassan, International Journal of Veterinary Science and Medicine, 6, 65-72 (2001)

12. B. Kumar, B.R. Maharana, A. Prasad, J.P. Joseph, B. Patel, J.S. Patel, Journal of Parasitic Diseases, 40(4), 1342-1346 (2016) 
13. K. Hatam Nahavandi, A.H. Mahvi, M. Mohebali, H. Keshavarz, S. Rezaei, H. Mirjalali, et al., Jundishapur Journal of Microbiology, 9(4), e34140 (2016)

14. A.C. Satish, K. Nagarajan, C. Balachandran, C. Soundararajan, R. Legadevi, International Journal of Livestock Research, 9 (02), (2019) DOI: 10.5455 / ijlr.20180603054413.

15. L. Oliveira de Macedo, M.A. Bezerra Santos, N.M. Marinho da Silva, G.M. Maciel do Rêgo Barros, L.C. Alves, A. Giannelli, R.A. Nascimento Ramos, G. Aparecida de Carvalho, Small Ruminant Research, 171, 37-41 (2019)

16. T.A. Ahmad, D.M. Tawfik, S.A. Sheweita, M. Haroun, L.H. El-Sayed, Trials in Vaccinology, 5, 38-47 (2016) DOI: 10.1016 / j.trivac.2016.03

17. K. Al-Habsi, R. Yang, U. Ryan, D.W. Miller, C. Jacobson, Veterinary Parasitology: Regional Studies and Reports, 9, 75-83 (2017) DOI: 10.1016 / j.vprsr.2017.05.001

18. A. Khodakaram-Tafti, M. Hashemnia, Revue de médecine vétérinaire (Toulouse), 167, 9-20 (2017)

19. I. Kyriánová, J. Vadlejch, I. Langrová, Science Agriculturae Bohemica, 48, 70-75 (2017)

20. L.M.R. Silva, F. Chávez-Maya, S. Macdonald, E. Pegg, D.P. Blake, A. Taubert, C. Hermosilla, Veterinary Parasitology, 248, 28-32 (2017) DOI: 10.1016 / j.vetpar. 2017.10.014 